Ann. Sci. forest., I975, 32 (4), 205-22I.

\title{
UTILISATION DES PHOTOGRAPHIES HÉMISPHÉRIQUES POUR LE CALCUL DE LA PERMÉABILITÉ DES COUVERTS FORESTIERS AU RAYONNEMENT SOLAIRE
}

\author{
II. - ÉTUdè EXPéRIMENTALE
}

\author{
M. DUCREY \\ Station de Sylviculture et de Production, \\ Centri national de Recherches forestières, I. N. R. A., \\ Champenoux, 54280 Seichamps
}

\section{RÉSUMÉ}

L'auteur rappelle tout d'abord brièvement la méthode utilisée pour calculer la perméabilité relative d'un couvert forestier au rayonnement solaire et l'éclairement énergétique sous ce couvert, à partir de photographies hémisphériques prises sous le feuillage.

Il compare ensuite les résultats obtenus à partir des photographies, à des mesares directes de rayonnement solaire faites sous différents peuplements forestiers.

Pour des éclairements relatifs supéricurs à 15-20 p. Ioo, les photographies hémisphériques donnent des résultats très proches des valeurs obtenues avec les pyranomètres.

Dans ces conditions, si on mesure le rayonnement global en plein découvert, on peut estimer avec une bonne précision le rayonnement global sous un peuplement, en utilisant uniquement des photographies hémisphériques.

Pour les éclairements relatifs inlérieurs à io p. ioo, les photographies hémisphériques sous. estiment l'éclairement réel. Il faut alors tenir compte du coefficient de transmission du feuillage et de la composition spectrale de la lamière.

\section{INTRODUCTION}

Ia mesure directe du rayonnement solaire pose de nombreux problèmes techniques, notamment en forêt. C'est pourquoi, quand les conditions expérimentales n'exigent pas ces mesures directes, il est préférable d'utiliser des méthodes indirectes, plus faciles à mettre en ouvre. C'est aussi pour cela que l'idée d'utiliser les photographies hémisphériques pour apprécier l'éclairement relatif sous les couverts végétaux est relativement ancienne. Depuis que Hill (I924) a décrit son objectif 
de $180^{\circ}$ d'angle de visée, nombreux sont les auteurs qui ont été tentés par cette méthode : Evans et CoOmbe (I959), Clark (I96I), ANDERson (I964), BECKER (I97 I) parmi d'autres. Sans utiliser ces photographies, Roussei. (I953) a essayé de calculer de manière théorique, l'éclairement relatif dans des trouées forestières de forme circulaire. Mais ces différents travaux n'ont rendu compte que de manière très schématique de l'origine et de l'intensité du rayonnement solaire incident; c'est pourquoi ils n'ont eu que des applications pratiques très limitées.

Compte tenu de l'intérêt de cette technique en recherche forestière si son utilisation pouvait être faite à grande échelle, nous nous sommes très rapidement intéressé aux photographies hémisphériques (D)UCrey, I970). Nous avons essayé de reproduire le plus fidèlement possible le climat radiatif local en prenant en compte les variations instantanées, journalières et saisonnières du rayonnement global (DUCREY, I974, I975).

Parallèlement à nos travaux, Bonhomme (I974) a porté un effort similaire dans le cas des cultures agronomiques. Mais comme il s'est intéressé surtout à des mesures d'éclairement relatif sous des couverts homogènes (1) il n'a pas été obligé d'approfondir les questions concernant 1a simulation du climat radiatif.

Nous avons, quant à nous, étudié le cas général des couverts hétérogènes que l'on retrouve souvent sous les peuplements forestiers et dans des traitements sylvicoles classiques : éclaircies, coupes de régénération, plantations sous abri, en coupes rases par bandes... Il est en effet très important en sylviculture de connaitre 1'influence de chaque opération sylvicole sur la répartition de l'énergie dans les houppiers (impact d'une éclaircie sur la productivité primaire) et au niveau du sol (croissance des plantations et régénérations). Nous avons expliqué en détail le principe de notre méthode dans deux publications récentes (DUCREy, I974, I975) c'est pourquoi nous n'en dirons que quelques mots au paragraphe suivant.

Dans cet article, nous voulons comparer les éclairements relatifs calculés d'après les photographies hénisphériques aux valeurs réelles de rayonnement global mesuré aux mêmes emplacements. Ce genre de comparaison a donné des résultats satisfaisants pour les couverts agronomiques (Bonhomms, 1974) mais il n'y a pas eu de travail identique pour les converts forestiers, du moins pas avec des méthodes techniquement et théoriquement très élaborées. Nous avons donc entrepris cette comparaison pour savoir si oui ou non la technique pouvait être substituée dans certains cas aux mesures directes de rayonnement global et, si oui, dans quelles conditions précises.

\section{II. - DISPOSITIFS FXPÉRIMENTACX}

Nous avons profité pour faire ce travail, de plusicurs dispositifs expérimentaux d'étude des microclimats forestiers, dans lesquels nous mesurons le rayonnement global. Pour pouvoir effectuer des comparaisons, nous avons pris des photographies hémisphériques à l'emplacement de tous les pyranomètres. Ces dispositifs sont au nombre de trois.

(1) Couvert homogène : couvert dans lequel la répartition des tronees est statistiquencit indepen dante de l'azimut, par opposition à couvert hétérogène pour lequel cette hypothèse n’ect pas vérifíe. 


\section{I. - Profil vertical dans un peupleinent de Hêtre}

Le peuplement est une futaie de Hâtre âgée de 80 ans et haute en moyenne de 25 m située en forêt domaniale d'Amance. Le profil vertical de rayonnement a été effectué grâce à huit niveaux de mesure répartis tout au long d'une tour métallique qui dépasse le peuplement. La référence de plein découvert est donnée par un pyranomìtre Kipp et Zonen situé au sommet de la tour.

\section{Répartition horizontale sous un peuplement de Hêtre}

Le même peuplement a servi à étudier la répartition horizontale du rayonnement solaire. Vingt points de mesure ont été répartis suivant un quadrillage régulier de ro màtres de côté. Le peuplement, bien que considéré comme homogène comporte quelques "microslairières" dues au passage récent d'une éclaircic.

\section{Répartition du rayonnement global dans une plantation en coste rase par bante}

(Forêt domaniale de Pelenq (Var) (1))

Dans le taillis d'origine des bandes de 5 mètres de largeur ont été coupés à blanc. Elles sont séparées par des bandes ayant aussi 5 mètres de largeur et dans lesquelles on a laissé subsister le taillis. Des mesures d'éclairement ont été faites en divers endroits caractéristiques dans ces bandes et en plein découvert.

Les mesures ont été faites à l'aide de pyranomètres linéaires (type I. N. R. A.) et de pyranomètres Kipp et Zonen; ces derniers étant utilisés pour les références de plein découvert. Les données sont récoltées grâce à une centrale de mesure Schlumberger (UTD) à la calense de trois mesures par seconde et de un cycle de mesures toutes les cinq minutes.

A chaque point de mesure, nous avons pris des photographies hémisphériques par temps parfaitement couvert et en orientant les photographies par rapport au Nord.

\section{III. - PRINCIPE; DE I,A MÉTHODE UTILISÉE}

La perméalilité relative d'un feuillage donné est égale au rappoit de deux valeurs de rayonnement global calculées mathématiquement à partir de formules sami-empiriques. Le numérateur représente le rayonnement global qui atteint le point de mesure après avoir traversé les trouées du feuillage. Le dénominateur représente le rayonnement global qui atteindrait ce point en l'absence de tout obstacle (voir DUCREY, I974).

La présence des obstacles est quantifiée par la probabilité de voir le cial dans une direction donnée ou par le pourcentage de ciel qui est obstrué par le feuillage dans un "carré "éléməntaire de ciel. On utilise alors le torme de fréquence des troués.

$$
\text { I. Perméabilité relative au rayonnement solaire direct: I's }
$$

On la calcule directement, à l'échelle de la journée, par l'équation :

$$
\mathrm{P}_{s}(\mathrm{~J})=\frac{\Sigma, S_{r}\left(\mathrm{~A}_{0}, h\right) \operatorname{ESD}(t, \mathrm{~J})}{\Sigma_{t} \operatorname{ESD}(t, \mathrm{~J})}
$$

dans laquelle :

ESD $(t, J)$ est l'éclairement solaire direct calculé à l'instant $t$ da jour J,

- $S_{r}\left(A_{0}, h\right)$ est la surface relative des taches de soleil; c'est en fait la fréquence des trouéss dans la direction du soleil déteminée par la hauteur angulaire $h$ et l'azimut $A_{0}$.

Pour un instant $t$ de la journée, le soleil étant alors dans la position $A_{0}$, $h$, la perméabilité au ravonnement solaire direct s'écrit bien simplement: $\mathrm{P}_{s}(t, \mathrm{~J})=\mathrm{S}_{r}\left(\mathrm{~A}_{0}, h\right)$.

(1) La forêt Domaniale de Peleng se trouve à $35 \mathrm{~km}$ au Nord-Onest de Draguignan, à une altitude voisine de 500 mètres. Située sur sol calcaire, elle est essentiellement constituée par un taillis de chène pubescent, hant en moyenne de 5 mètres. 


\section{2. - Perméabilité relative au rayonnement diffusé par ciel serein $\mathrm{P}_{a}$}

Le calcul en est un peu plus compliqué car, à chađ̛ue instant de la journée on doit tenir compte de la luminance de tous les points du cicl, chacun d'eux étant alors considéré comme une source de rayonnement diffus.

Pour chaque instant $t$ d'un jour donné $\mathrm{J}$, la perméabilité au rayonnement diffusé par ciel serein s'écrit :

arec:

$$
P_{d}(t, J)=\frac{\Sigma_{\theta}}{-\Sigma_{A} q(A, \theta) L(A, \theta) \sin \theta \cos \theta}
$$

- $q(\mathrm{~A}, \theta)$ : fréquence des trouées (mesurée sur photographies hémisphériques) dans la direction caractérisée par l'azimut A et la hauteur angulaire $\theta$,

- L $(\mathrm{A}, \theta)$ : luminance du point du ciel de coordonnées A et $\theta$.

Pour avoir la perméabilité moyenne sur un jour complet, il suffit d'intégrer çaqus terme du rapport en fonction de $t$.

\section{3. - Perméabilité relative au rayonnement global par ciel serein $\mathrm{P}_{g}$}

Le rayonnement global est la somme du rayonnement solaire direct et du rayonnement diffusé par le ciel. La perméabilité $\mathrm{P}_{g}$ est donc définie comme le rapport entre le rayonnement global calculé qui arrive effectivement au point de mesure ct le rayonnement global calculé pour un ciel parfaitement dégagé.

\section{4. - Perméabilité relative au vayonnement diffusé par ciel convert $\mathrm{P}_{c}$}

On la calcule exactement de la même manière que dans le cas du ciel screin. Mais la foiction qui définit la luminance est beaucoup plus simple: elle ne dépend pas, en particulier, de l'azimut.

Ces quatre types de perméabilités sont calculés pour chaque photographie hémisphérique à l'aide d'un programme de calcul sur ordinateur. Ce programme exécute les calculs heure par heure pour chaque jour que l'on choisit. Les saules données nécessaires, en dehors des tableaux $q(\mathrm{~A}, \theta)$ pour chaque photographie, sont :

- les coordonnées géographiques du lieu (latitude et longitude),

- les conditions atmosphériques moyennes (coefficient de trouble atmosphérique),

- et éventuellement, la pente et l'azimut de la surface (cas d'un terrain en pente).

Nous avons ensuite comparé les éclairements absolus et les perméabilités relatives obtenues grâce aux photographies hémisphériques, avec les valeurs corresponclantes mesurées réellement par les pyranomètres.

\section{IV. - RÉSULTATS}

Les résultats présentés portant tout d'abord sur la comparaison entre les éclairements relatifs obtenus par les deux méthodes. Puis nous traiterons ensuite des valeurs absolues d'éclairement obtenues grâce aux calculs effectués à partir des photographies hémisphériques.

\section{I. - Comparaison des éclairements relatifs journaliers}

Pour faciliter les comparaisons nous avons séparé les journées parfaitement claires et les journées complètement couvertes. Dans chaque figure, nous avons représenté en abcisse les éclairements relatifs et en ordonnée les perméabilités 

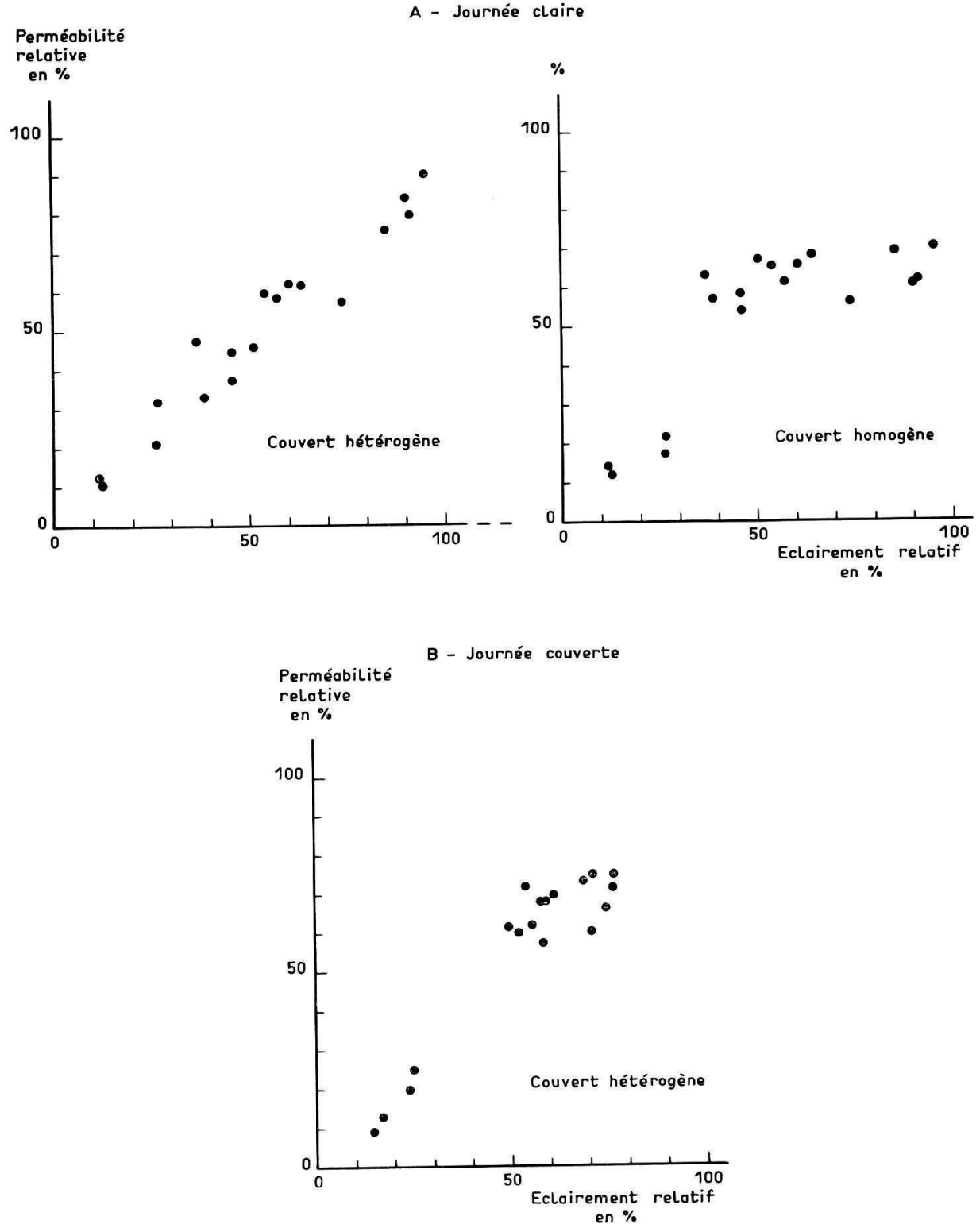

FIG. I. - Comparaison des valeurs journalières d'éclairement relatif et de perméabilité relative $\left(\mathrm{P}_{g}\right)$ à Pelenq (Var)

a) par ciel clair

à gauche : hypothèse de couvert hétérogène $(\mathrm{R}=0,97 \mathrm{I})$

à droite : hypothèse de couvert homogène ( $\mathrm{R}=0,77 \mathrm{I})$

b) par ciel couvert.

Compared daily values of irradiance ratio and relative permeability $\left(\mathrm{P}_{g}\right)$ at Pelenq (Var)

a) with a clear sky left : heterogeneous canopy $(\mathrm{R}=0,97 \mathrm{I})$ right : homogenous canopy ( $R=0,77 \mathrm{I})$

b) with an overcast sky. 

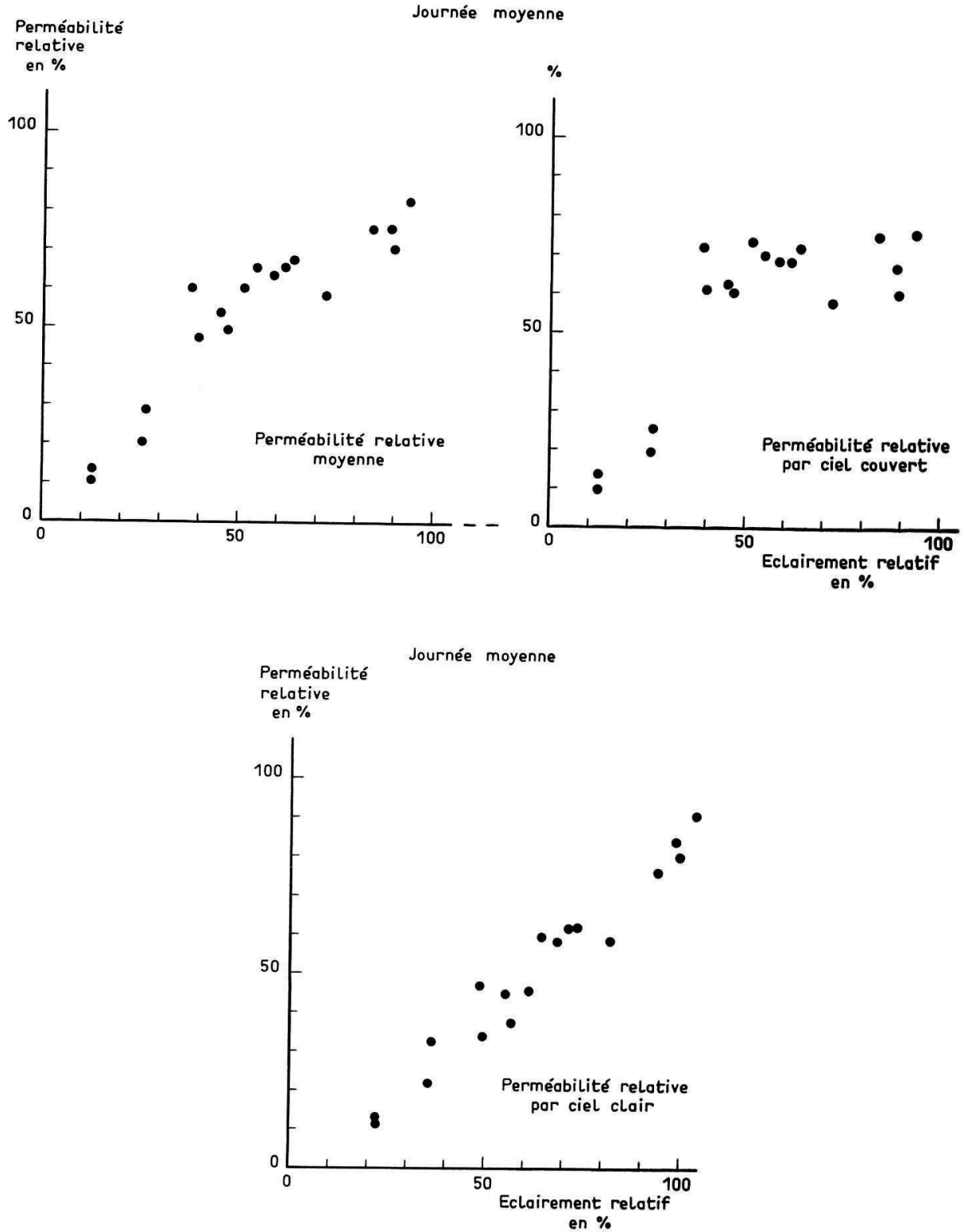

Fic. 2. Comparaison entre l'éclairement relatif moven

(moyenne de 6 journées d'observation à Peleng) et différentes perméabilités pelatives

en haut à gauche : perméabilití relative moyenne $\left(\mathrm{P}_{g}+\mathrm{P}_{c}\right) / 2$

en haut à droite : perméabilité relative par ciel couvert $\mathrm{P}$

en bas : perméabilité relative par ciel clair $\mathrm{P}_{g}$

Comparison between the mean irradiance ratio

(the mean of 6 days' observation at Pelenq) and various relative permeabilites

upper left : mean relative permeability $\left(\mathrm{P}_{g}+\mathrm{P}_{c}\right) / 2$

upper right: relative permeability with an overcast sk $P_{c}$

bottom : relative permeability with a clear sliy $\mathrm{P}_{y}$ 
Perméabilité

relotive

en $\%$

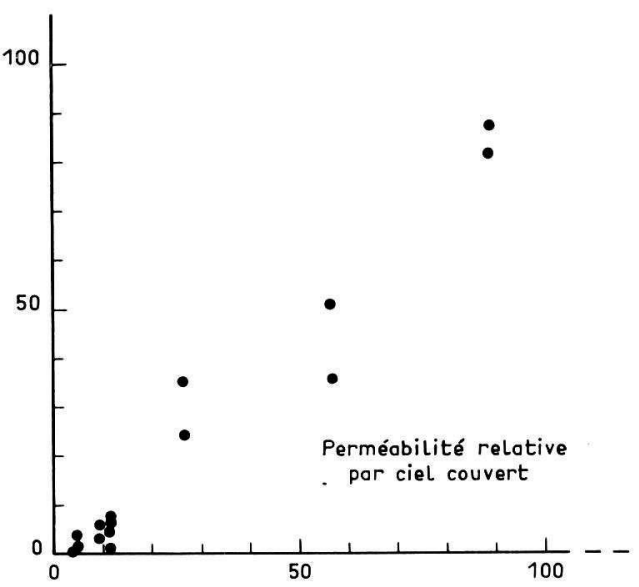

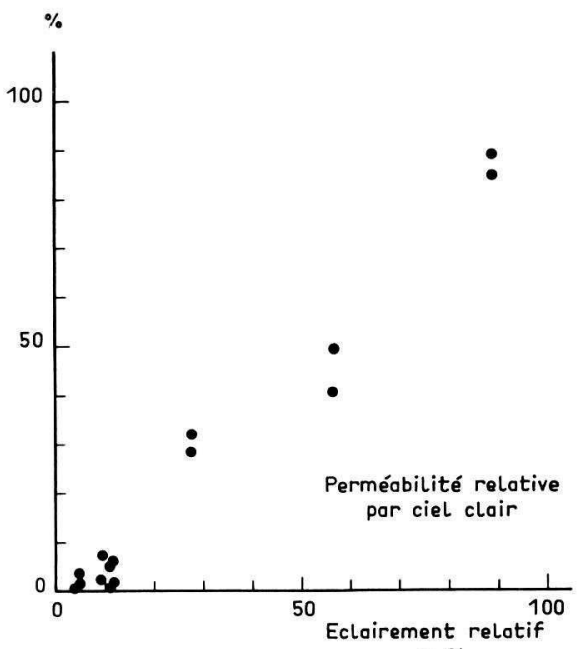

en $\%$

FIG. 3. - Comparaison des valeurs journalieres d'clairement relatif par ciel clair et de perméabilité relative dans un peuplement de hêtre (Forêt d'Amance)

à gauche : perméabilité relative par ciel couvert $\mathrm{P}_{c}$

a croite : perméabilité relative par ciel clair $\mathrm{P}_{g}$

Compared daily values of the irradiance ratio with a clear sky

and of the relative permeability under a beech stand (Forest of Amance, Meurthe-et-Moselle)

left : relative permeability with an overeast sky $P_{c}$

right : relative permeability with a clear sky $\mathrm{P}_{g}$

Journée claire

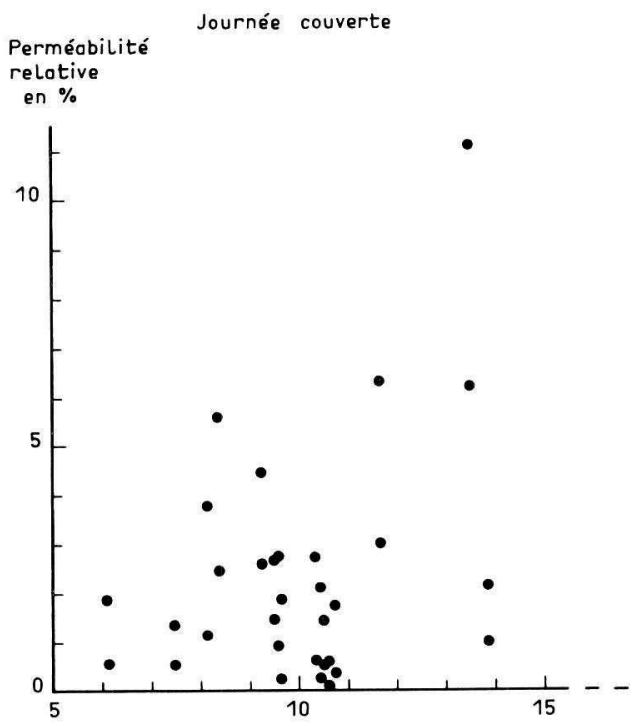

\section{Perméabilité}

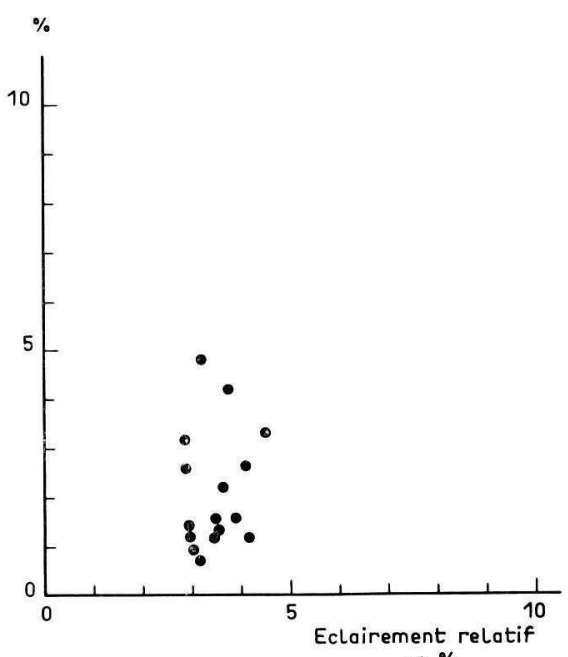

en $\%$

FIs. 4. Comparaison des éclairements relatifs et des perméabilités relatives sous un peuplement dense de hêtre (Forêt d'Amance)

a gauche : comparaison par ciel couvert

it droite : comparaison par ciel clair

Compared irradiance ratio and relatize permeability

under a dense beech stand (Forest of Amance, Meurthe-et-Moselle)

left : comparison with an overcast sky

riwht : comparison with a clear sky 
relatives. Les photographies hémisphériques donneront donc les meilleurs résultats quand les points expérimentaux seront alignés sur la première bissectrice.

La figure I a montre pour une journée claire moyenne à Pelenq la correspondance entre éclairements relatifs (mesurés) et perméabilités relatives (calculées). L'ajustement est très bon (coefficient de corrélation de $0,97 \mathrm{I}$ ) quand on mesure la fréquence des trouées pour chaque " carré » de ciel : $q(\mathrm{~A}, \theta)$. Mais comme jusqu'à maintenant seul le cas des couverts homogènes avait été étudié théoriquement (Bonhomme, 1974) nous avons essayé d'adopter cette hypothèse (qui est manifestement inacceptable dans le cas de Pelenq) et nous avons calculé les perméabilités relatives en faisant intervenir $q(\theta)=\Sigma_{\mathrm{i}} q(\mathrm{~A}, \theta)$ an lieu de $q(\mathrm{~A}, \theta)$. Dans cette hypothèse l'ajustement des valeurs calculées aux valeurs mesurées est beaucoup moins bon $(\mathrm{R}=0,77 \mathrm{I})$. Le gain de précision obtenu en remplaçant $q(\theta)$ par $q(\mathrm{~A}, \theta)$ est donc très appréciable.

Pour une journée de temps à peu près uniformément couvert, mais cependant assez lumineux (rayonnement global égal à $\mathrm{I} 4 \mathrm{MJ} \mathrm{\textrm {m } ^ { - 2 }}$ contre $23,3 \mathrm{MJ} \mathrm{m}^{-2}$ par temps clair), la figure I $b$ montre une plus grande dispersion des points surtout pour les forts éclairements relatifs. La droite de régression montre que quand la perméabilité relative est nulle, l'éclairement relatif est encore de $3 \mathrm{p}$. Ioo.

Pour la durée totale des mesures de Pelenq, nous avons observé trois journées claires, deux journées nuageuses et une journée couverte. Nous avons aussi pu mesurer un éclairement relatif moyen pour chacun des dix-huit points expérimentaux. Ces données ont été comparées aux perméabilités relatives calculées par temps clair et par temps couvert et à une perméabilité relative moyenne (moyenne arithmétique des deux perméabilités précédentes. Les résultats obtenus (fig. 2) sont les meilleurs pour la perméabilité relative par temps clair.

Les résultats obtenus ci-dessus correspondent à une gamme complète d'éclairements relatifs allant de quelques pour cent à près de cent pour cent. On retrouve des résultats analogues (fig. 3) pour des profils radiatifs verticaux effectués dans une futaie de Hêtre. Mais dans ce profil, nous constatons que les perméabilités correspondant à des éclairements relatifs inférieurs ou voisins de to p. Ioo sont systématiquement sous-estimées. On retrouve ce fait aussi bien par temps convert que par temps clair.

Cette tendance des photographies hémisphériques à la sous-estimation se trouve généralisée pour les faibles éclairements relatifs. La figure 4 met en évidence ce phénomène dans le cas de l'éclairement relatif mesuré sous un peuplement fermé de Hêtre. On remarquera aussi qu'il n'y a pratiquement pas de corrélation entre perméabilité relative et éclairement relatif.

\section{2. - Comparaison des éclairements relatifs horaires}

Pour nous rendre compte de la précision de notre méthode et pour essayer de savoir si nous pouvions nous servir de nos calculs pour estimer des éclairements à une échelle de temps inférieure à la journée, nous avons effectué des comparaisons horaires (notre programme de calcul donnant directement les perméabilités relatives horaires). Cette comparaison est possible grâce au dispositif expérimental de Pelenq dans lequel nous avons calculé les éclairements horaires théoriques à partir de notre programme de calcul par ordinateur. 
Dans un premier temps, nous avons comparé entre eux les différents éclairements (ou perméabilités) relatifs horaires. 'Tout d'abord nous avons tracé pour chacun des dix-huit points expérimentaux les droites de régression entre les éclairements relatifs horaires et les perméabilités relatives horaires (I3 couples de valeurs entre 7 et I9 heures). Puis nous avons calculé les dix-huit coefficients de corrélation correspondants. La figure 5 a montre la répartition de ces coefficients de corrélation en fonction de l'éclairement relatif journalier calculé pour chaque point de mesure.

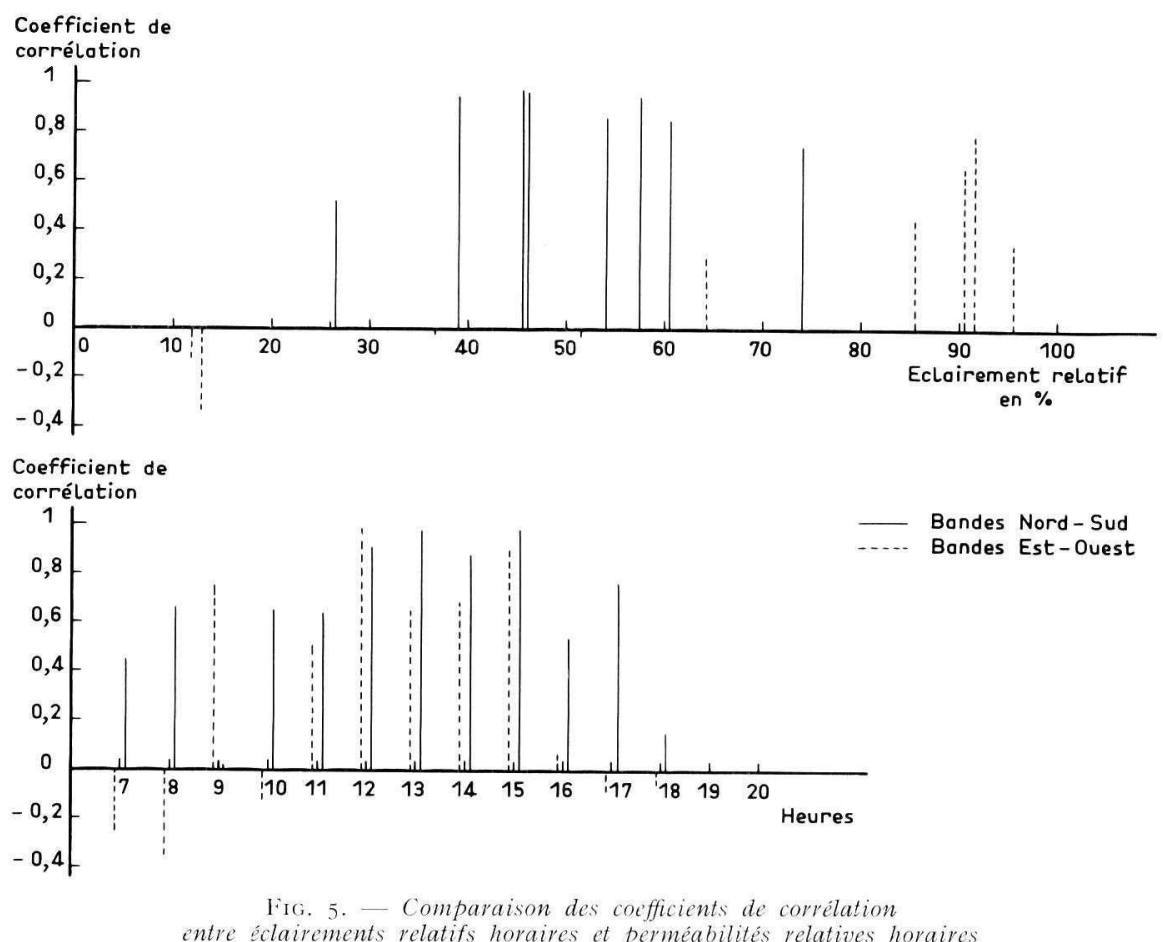

a) en haut : évolution des coefficients de corrélation en fonction de l'éclairement relatif journalier de chaque point de mesure,

b) en bas : évolution des coefficients de corrélation en fonction de l'heure du jour.

Compared correlation coefficients of hourly irradiance ratio and hourly relative permeability

a) top : evolution of the correlation coefficients versus daily irradiance ratio at each measurement point,

b) botton : evolution of the correlation coefficients versus the time of the day.

Il apparaît que la valeur des coefficients de corrélation angmente avec l'éclairement relatif des stations étudiées et que les stations situées dans les bandes d'orientation Nord-Sud donnent de meilleurs résultats que celles des bandes Est-Ouest. Ceci est dî au fait que dans le premier cas les situations horaires sont plus tranchées : pyranomètre franchement à 1'ombre ou au soleil, tandis que dans le second cas beaucoup de situations intermédiaires, donc d'imprécisions dans les estimations, sont possibles.

Nous avons ensuite tracé pour chaque heure de la journée les droites de régression entre éclairement et perméabilité correspondant à chaque point de mesure. La figure $5 b$ montre aussi comment varient les coefficients de corrélation corres- 
pondants en fonction de l'heure. On observe, pour les mêmes raisons que précédemment que ces derniers sont plus élevés dans les bandes Nord-Sud que dans les bandes Est-Ouest et que les valeurs les plus fortes sont au voisinage de midi.

Dans tous les cas, à des coefficients de corrélation élevés, correspondent des droites de régression très voisines de la première bissectrice. Les estimations horaires par photographies hémisphériques ne sont donc pas biaisées par rapport aux mesures directes.

\section{3. - Comparaison des éclairements énergétiques journatiers}

Notre méthode est la première qui utilise pour le calcul de la perméabilité relative, des lois théoriques de variation du rayonnement global qui sont très proches de la réalité. C'est pourquoi il nous a semblé intéressant de comparer les éclairements théoriques (horaires ou journaliers) calculés pour chaque point de mesure, aux éclairements mesurés réellement par des pyranomètres.

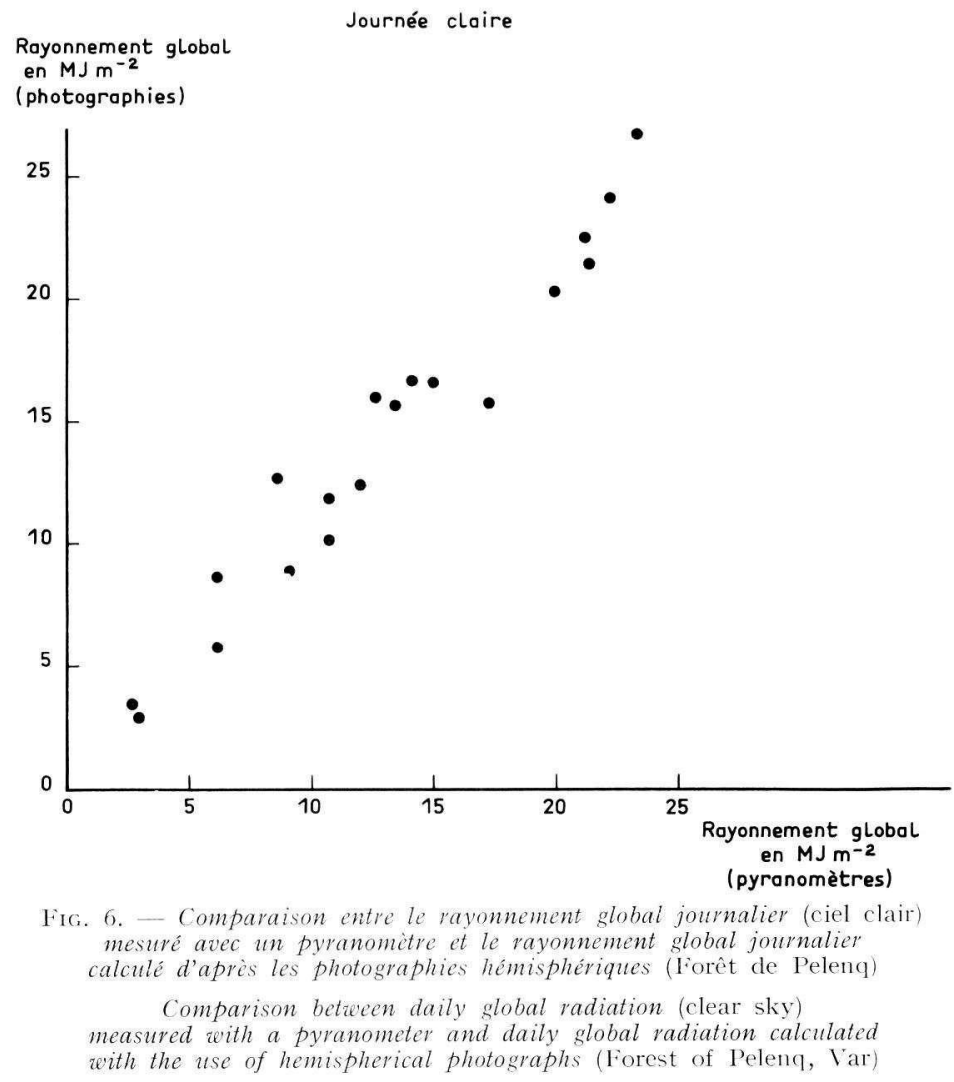

Nous avons reporté (fig. 6) les éclairements journaliers (en MJ $\mathrm{m}^{-2}$ ) calculés pour chacun des dix-huit points expérimentanx de Pelenq, d'après les données pyranométriques recueillies pendant trois jours clairs. En ordonnée nous avons représenté les éclairements théoriques calculés d'après notre programme de calculs pour le jour $\mathrm{J}=240$ correspondant à la date de nos mesures, et en prenant un 
coefficient de trouble atmosphérique $\mathrm{T}=3,25$. Cette valeur a été choisie d'après les indications de Dogniaux (I973) préconisant cette valeur dans le cas d'un site rural (absence de pollution) et ayant un air assez peu humide (Pelenq se trouve en Haute-Provence). Mais ce coefficient s'est cependant avéré un peu trop faible ce qui explique les différences de ${ }_{5}$ p. Ioo que l'on observe dans toutes les comparaisons notamment dans la pente de la droite de régression de la figure 6 . Ceci mis à part, la comparaison entre les éclairements énergétiques donne de très bons résultats qui sont à rapprocher de ceux obtenus avec les éclairements relatifs journaliers.

\section{4. - Comparaison des éclairements énergétiques horaires}

Nous avons représenté sur la figure 7, l'évolution au cours de la journée, des éclairements calculés et des éclairements mesurés, pour un certain nombre de points d'expérimentation. Mis à part le fait que les valeurs calculées sont en moyenne I5 p. IOo supérieures aux valeurs mesurées, on trouve un bon parallélisme entre
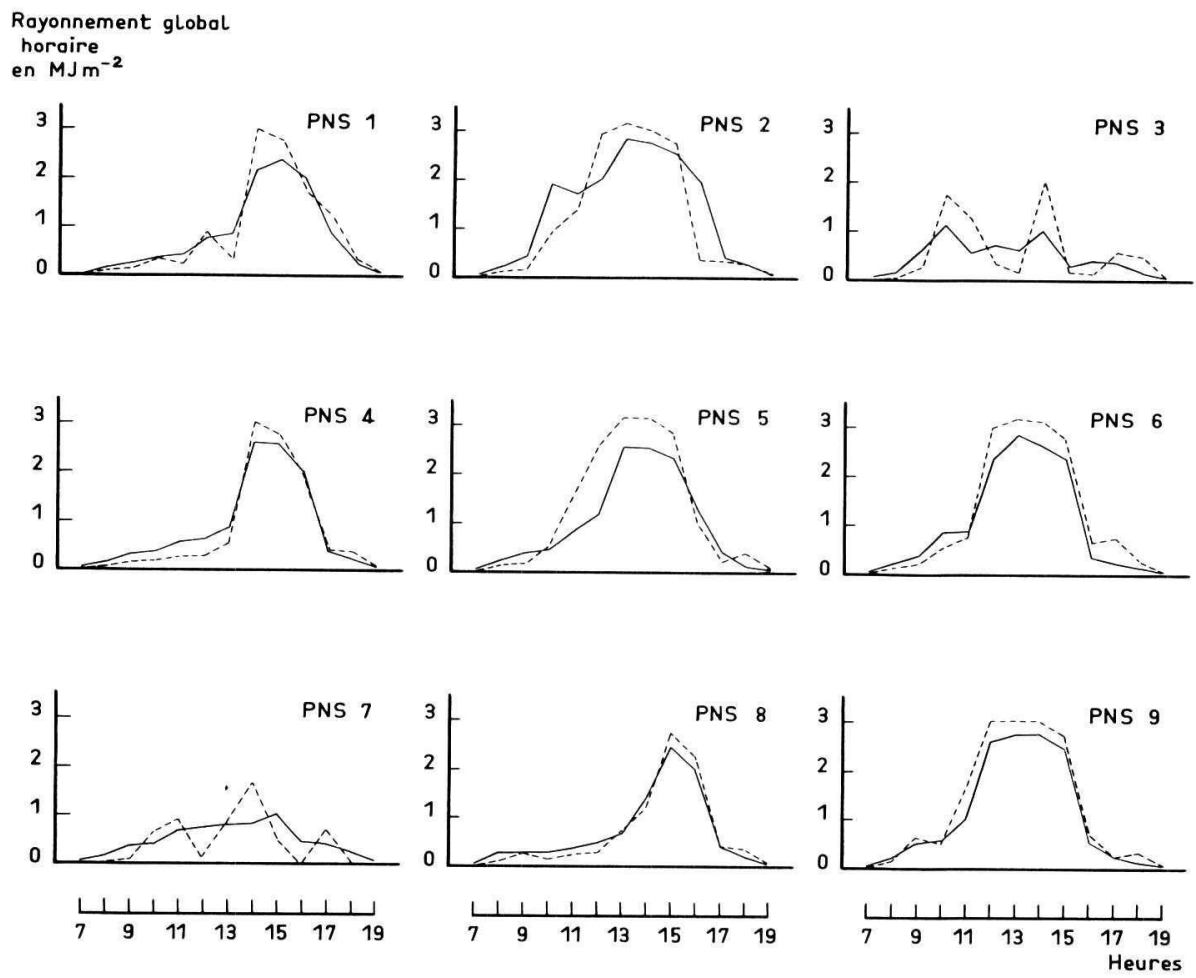

FIG. 7. - Evolution du rayonnement global au cours de la journée pour différents points de mesure (Forêt de Pelenq)

- mesures directes par pyranomètres

..... valeurs calculées par photographies hémisphériques

Daily evolution of the global radiation

at various measurement points (Forest of Pelenq, Var)

- direct measures from pyranometers

..... values calculated from hemispherical photographs 


\section{TABI,EAU I}

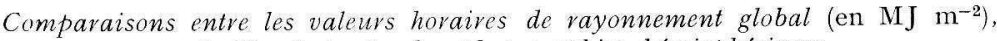
calculées à partir des photographies hémisphériques

et mesurées par des pyranomotres, pour différents points de mesure

Comparison between hourly values of the global vadiation (in $\mathrm{MJ} \mathrm{m} \mathrm{m}^{-2}$ ), calculated from hemispherical photographs, and measured with pyranometers

\begin{tabular}{|c|c|c|c|}
\hline Station & \multicolumn{2}{|c|}{$\begin{array}{l}\text { Droite de régression } \\
\qquad y=a+b x\end{array}$} & \multirow{2}{*}{$\begin{array}{c}\begin{array}{c}\text { Coefficients } \\
\text { de corrélation } \\
\mathrm{R}\end{array} \\
0,998\end{array}$} \\
\hline Plein dćcouvert & $-0,0,21$ & 1,153 & \\
\hline PNS 1 & $-0,111$ & 1,248 & 0,975 \\
\hline PNS 2 & $-0,187$ & $1,0 \prime 13$ & 0,879 \\
\hline PNS 3 & $-0,026$ & 1,450 & 0,719 \\
\hline PNS 4 & $-0,150$ & 1,127 & 0,983 \\
\hline PNS 5 & 0,009 & 1,264 & 0,951 \\
\hline PNS 6 & 0,001 & 1,165 & 0,985 \\
\hline PNS $\%$ & $-0,021$ & 0,986 & $0,622(1)$ \\
\hline PNS 8 & $-0,0 S^{\prime}$ & 1,099 & 0,989 \\
\hline PNS 9 & 0,036 & 1,140 & 0,992 \\
\hline PEW 1 & 0,336 & 1,275 & 0,977 \\
\hline PEW 2 & $-0,105$ & 1,203 & 0,929 \\
\hline PEW 3 & $-0,090$ & 1,697 & $0,7 \pm 8$ \\
\hline PEW 4 & $\longrightarrow 0,290$ & 1,180 & 0,968 \\
\hline PEW 5 & $-0,150$ & 1,120 & 0,890 \\
\hline PEII 3 & 0,181 & 1,120 & 0,582 \\
\hline PEII 7 & $-0,004$ & 1,009 & $0,60.3$ \\
\hline PEW \& & $-0,095$ & 1,111 & 0,992 \\
\hline PEW 9 & $0,87 . ;$ & 0,113 & $0,17:$ \\
\hline
\end{tabular}

(1) Les valeurs soulignées correspondent aux situations où la corrélation entre valeurs calculces et valeurs mesurées est mauvaise. (The underlined values represent points where the correlation between calculated and measured values is poor). 
les 2 types de courbes, excepté pour les points PNS 3 et PNS 7 qui correspondent à des emplacements sous taillis fermé, donc à des éclairements faibles.

Le tableau I sur lequel nous avons fait figurer les équations des droites de régression linéaire des éclairements calculés en fonction des éclairements mesurés montre la très bonne relation qui existe entre les deux méthodes d'estimation des éclairements horaires. L'examen des résultats montre que les droites qui ont des coefficients de corrélation élevés (supérieurs à 0,9 ) ont des pentes qui diffèrent de moins de io p. Ioo de la courbe de référence obtenue avec le plein découvert. Les relations entre éclairements énergétiques sont plus étroites que celles obtenues avec les éclairements relatifs ainsi que le montre la comparaison de la figure 5 a et du tableau I.

\section{V. - DISCUSSION}

Dans le paragraphe précédent nous avons présenté de nombreux résultats que nous allons tenter maintenant de regrouper et d'analyser, en essayant de voir dans quels cas les estimations par photographies hémisphériques peuvent avantageusement remplacer les mesures directes de rayonnement.

Les valeurs journalières d'éclairement ont été étudiées du point de vue des éclairements relatifs et du rayonnement global en séparant les journées claires des journées couvertes. Les perméabilités relatives journalières sont dans le cas de Pelenq très étroitement liées aux éclairements relatifs journaliers. La relation est meilleure par journée claire que par journée couverte. Le fait que la journée couverte ait été très lumineuse (rayonnement global atteignant $60 \mathrm{p}$. Ioo de celui d'une journée claire) donc en réalité assez différente du ciel standard uniformément couvert explique les moins bons résultats obtenus dans ce cas. Les observations faites dans la hêtraie montrent de bonnes relations entre photographies et pyranomètres quand 1'éclairement relatif dépasse Io à 20 p. Ioo, ce qui est le cas pour les niveaux supérieurs du profil vertical.

Les résultats médiocres obtenus pour les niveaux inférieurs et au niveau du sol, aussi bien par ciel clair que par ciel couvert, peuvent s'expliquer de deux manières complémentaires. La figure 6 montre que les perméabilités relatives sous-estiment l'éclairement relatif et que les deux données ne sont pas corrélées entre elles.

La sous-estimation est dûe au fait que, avec les photographies hémisphériques, on considère uniquement le rayonnement qui passe directement à travers les trouées du feuillage. Le rayonnement diffusé et retransmis par les fetilles n'est pas pris en compte dans le calcul de la perméabilité relative. Bonhomme (I974) donne une méthode de calcul assez complexe permettant de tenir compte de cette fraction du rayonnement solaire. Nous pensons dans l'avenir l'intégrer dans nos calculs de la manière suivante :

Une surface élémentaire unitaire de ciel située au point $A, \theta$ est composée d'une surface $q(\mathrm{~A}, \theta)$ de trouées qui laisse passer directement le rayonnement solaire, et d'une surface I $-q(\mathrm{~A}, \theta)$ de feuillage. Ce feuillage a lui-même un certain coefficient de transmisssion $\mathrm{K}(\mathrm{F})$, fonction de l'indice foliaire total du peuplement $\mathrm{F}$. Cette fonction pourra être calculée soit expérimentalement à partir de données mesurées 
d'indices foliaires et d'éclairement relatif sous différents peuplements, soit théoriquement à partir des calculs de BonHomme.

Il faudrait en fait considérer séparément le visible et l'infrarouge. Dans le visible, le facteur d'absorption des feuilles est suffisamment élevé pour que l'on puisse, en première approximation, les assimiler à des corps opaques ( $\mathrm{K}(\mathrm{F})$ négligeable) et utiliser de ce fait, directement les résultats obtenus avec les clichés. L'infrarouge, par contre est peu absorbé par les feuilles et il faut alors tenir compte de $\mathrm{K}(\mathrm{F})$. Comme les pyranomètres I. N. R. A., sont sensibles à la fois au visible et à l'infrarouge, ceci permet d'expliquer certains résultats (fig. 4).
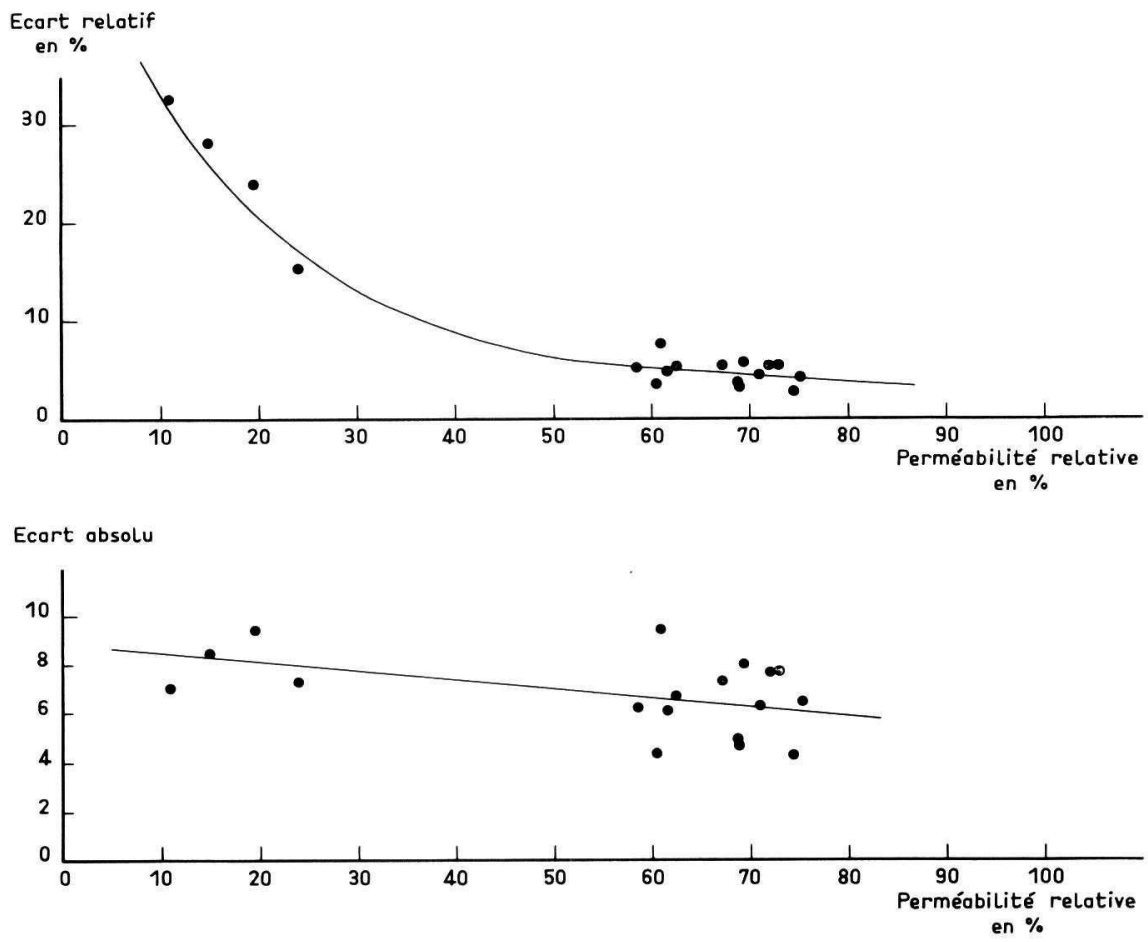

FiG. 8. - Comparaison des erreurs absolues et relatives

sur le calcul de la perméabilité (erreurs dues aux différences de prise de vue) en fonction des valeurs moyennes de perméabilité relative $P_{c}$

Compared absolute and relative errors on the calculation of the permeability (errors due to differences in brightness when taking the photograph) versus mean values of the relative permeability $P_{c}$

L'absence de corrélation entre éclairement et perméabilité pour les faibles pourcentages (inférieurs à Io p. Ioo) est dûe à des erreurs de temps d'exposition au moment de la prise des photographies hémisphériques. Ces erreurs sont imputables aux différences de luminosité d'un point à l'autre du ciel même par temps couvert. A Pelenq nous avons pris six photographies par point d'expérimentation à différents moments par temps couvert.

La figure 8 montre pour les perméabilités relatives par temps couvert, l'évo- 
lution des erreurs expérimentales en fonction de la perméabilité relative calculée. Les écarts absolus autour de la moyenne sont de $\pm 3,43$ et ont tendance à augmenter très légèrement quand la perméabilité est faible. Par contre les écarts relatifs passent de $\pm 5 \mathrm{p}$. Ioo pour des perméabilités élevées à près de $\pm 30 \mathrm{p}$. Ioo pour des perméabilités relatives de l’ordre de Io à 20 p. IOO. La précision des estimations faites sous convert fermé est donc très médiocre et c'est dans le domaine de la prise de vue qu'il faudra chercher des améliorations.

En ce qui concerne les valeurs théoriques journalières de rayonnement global, nous avons utilisé dans les calculs un coefficient de trouble atmosphérique $\mathrm{T}=3,25$ qui donne des valeurs I5 p. Ioo supérieures à celles mesurées réellement. Ce biais peut être éliminé en prenant pour T une valeur voisine de 4 à 4,5 . Cette nouvelle valeur n'est pas invraisemblable car au moment précis des expériences le ciel était d'un bleu très pâle ce qui indique que la quantité de vapeur d'eau dans l'atmosphère était très élevée. Sur le plan pratique on peut adopter la démarche suivante : pour une expérience donnée on mesure le rayonnement global journalier en plein découvert et on calcule le coefficient de trouble correspondant. On se sert ensuite des photographies hémisphériques pour calculer l'éclairement énergétique journalier de chaque point de mesure. D'après les résultats précédents, on sait que la précision sera très bonne pour les stations recevant une fraction importante du rayonnement incident (en tout cas supérieure à I5-20 p. IOO). C'est un cas que 1'on retrouve dans beaucoup d'interventions sylvicoles : éclaircies, coupes d'abri, coupes de régénération, coupes rases par bandes, tronées, clairières ou lisières. Seul le cas des peuplements fermés, donc à éclairement relatif faible, se prête mal aux estimations par photographies hémisphériques.

L'intérêt de ces résultats nous a conduit à étudier ce qui se passe au niveau des sommes horaires d'éclairement et des perméabilités relatives horaires. Sur la figure 7 , on peut voir que les erreurs absolues sur les estimations horaires sont à peu près du même ordre de grandeur quel que soit le point de mesure et l'heure de la journée ; ces erreurs étant bien entendu liées à l'erreur de mesure de $q(\mathrm{~A}, 0)$ sur les photographies hémisphériques. Ceci explique les résultats de la figure 5 sur les perméabilités horaires à savoir que les meilleures corrélations sont observées pour les points ayant un éclairement relatif élevé et pour les heures au milieu de la journée où la part du rayonnement solaire direct est prépondérante. Ceci explique aussi que les ajustements que l'on obtient avec les éclairements absolus sont meilleurs qu'avec les éclairements relatifs. Le tableau I montre des coefficients de corrélations élevés ce qui prouve que, même au niveau horaire, la précision des modèles de répartition de l'éclairement dans le ciel (utilisés dans nos calculs sur ordinateur) est suffisante. Ceci est dî̀ à la bonne superposition entre ces modèles (surtout au niveau de la trajectoire apparente du soleil) et les tableaux de fréquence de trouées $q(\mathrm{~A}, \theta)$. On a donc intérêt au moment de la prise des photographies à avoir le maximum de précision dans 1'orientation des clichés pour que cette superposition soit la meilleure possible.

İn ce qui concerne la fréquence des trouées, les différents auteurs ont toujours mesuré la fréquence moyenne pour une hauteur angulaire donnée $q(\theta)$ car les études ont porté sur des couverts homogènes. En introduisant la notion de fréquence ponctuelle pour un azimut et une hauteur angulaire donnés $q(\mathrm{~A}, \theta)$, nous avons apporté une amélioration très sensible des estimations par photographies hémis- 
phériques dans le cas des couverts hétérogènes. Ein effet, on voit sur la figure I que, pour les mêmes points de mesure, la corrélation entre perméabilité et éclairement passe de $0,77 \mathrm{I}$ à $0,97 \mathrm{I}$ quand on mesure $q(\mathrm{~A}, \theta)$ au lieu de $q(\theta)$.

\section{VI. - CONCLUSION}

Nous avons essayé de montrer au cours de cet article ce que nous pouvions attendre de l'utilisation en Forêt de la technique des photographies hémisphériques.

En ce qui concerne les microclimats radiatifs, nous avons très sensiblement amélioré cette technique en réalisant des modèles fonctionnels qui tiennent compte de l'énergie émise par chaque point du ciel et qui nécessitent de mesurer la fréquence des trouées de manière ponctuelle $q(\mathrm{~A}, \theta)$.

I'utilisation de cette technique améliorée nous a conduit aux deux conclusions suivantes. Les photographies prises sous des peuplements fermés, dont l'éclairement relatif est ordinairement inférieur à Io p. Ioo, ne donnent qu'une très médiocre appréciation de l'éclairement relatif réel. Les améliorations que nous pensons apporter prochainement sont d'ordre théorique (prise en compte du rayonnement diffusé et retransmis par le feuillage) et d'ordre technique (perfectionnement de la prise de vue et du traitement des clichés).

Par contre les résultats obtenus dans des peuplements non fermés sont très satisfaisants. Dans ce cas les deux sources d'erreurs signalées précédemment ont un poids relatif beaucoup plus faible car l'éclairement relatif est alors supérieur à I 5-20 p. Ioo. C'est ainsi que peuvent être estimés avec une précision suffisante pour beaucoup d'études les éclairements relatifs journaliers et même horaires pour les différents types de temps caractéristiques (temps clair et temps uniformément couvert).

En prenant la précaution de mesurer avec un pyranomètre le rayonnement global en plein découvert, il est même possible d'avoir une excellente estimation des énergies horaires et journalières arrivant à chaque point de mesure.

On peut ainsi envisager des études complètes de microclimatologie du rayonnement solaire avec l'aide des seules photographies hémisphériques et d'un pyranomètre de référence. Cette dernière conclusion est particulièrement intéressante quand on pense que la plupart des opérations sylvicoles dans les peuplements adultes ont pour but d'ouvrir ces peuplements (coupes d'abri, coupes de régénération, coupes rases par bandes...), notre méthode permettant alors de chiffrer les quantités d'énergie mises à la disposition des jeunes plantations ou des régénérations.

$$
\text { Reçu pour publication en août } 19 \% 5 \text {. }
$$

\section{SUMMARY}

U'TILIZATION OF HEMISPHERICAL, PHOTOGRAPHS

TO CAI,CULA'TE SOIAR RADIATION PERMEABIIITY OF FOREST CANOPIES.

II. - EXPERIMENTAI, STUDY

The author briefly recalls the method used for calculating the relative permeability of a forest canopy to solar radiation, and the irradiance under this canopy, based on hemispherical photographs taken from the ground. 
He then compares the results obtained with the photographs to direct measures of the solar radiation under various forest stands.

For irradiance ratio over $15-20$ p. IoO, the hemispherical photographs give results very similar to those obtained with pyranometers.

In these conditions, if we measure the global radiation in an open aera, we can estimate with a good precision the global radiation under a canopy, with the sole use of hemispaerical photographs.

For irradiance ratio under io p. Ioo, the hemispherical photographs under-estimate the actual irradiance. We must then under-account the transmittance of the foliage and the spectral composition of the light.

\section{ZUSAMMENFASSUNG}

\section{DIE; VERWENDUNG VON HEMISPHÄRISCHEN WEITWINKEIAUENAHMEN \\ ZUR BERECHNUNG DER STRAHLUNGSDURCHL̈̈SSIGKEIT

\begin{abstract}
DES KRONENDACHES IN WALDBESTANDEN.
\end{abstract}

\section{II. - EXPERIMENTEILE UNTERSUCHUNG}

Der Autor wicderholt vorerst die Methodik zur Berechnung der relativen Durchlässigkeit des Kronendaches und der Strahlungsenergie im Bestand an Hand von hemisphärischen Weitwinkelaufnahmen.

Anschliessend werden dic Ergebnisse mit direkten Strahlungsmessungen in verschiedenen Beständen verglichen.

Bei relativen Durchlässigkeiten von über I 5 bis 20 p. Ioo ergibt sich cine sehr gute Übereinstimmung mit den Pyranometermessungen.

Wenn die Globalstrahlung auf einer Freifläche gemsssan wird, kunn unter diesen Voraussetzungen die Globalstrahlung im Bestand ausschliesslich an Hand von homisphärischen Weitwinkelaufnahmen mit grosser Genauigkeit berechnet werden.

Bei Durchlässigkeiten unter ro p. roo wird die tatsächliche Globalstrahlung durch die Weitwinkelaufnahmen unterschätzt. Es müssen daher der Transmissionskoeffizient dər Blätter und die spektrale Zusammensetzung der Strahlung mit berücksichtigt wardon.

\section{RÉFÉRENCES BIBLIOGRAPHIQUES}

Axpr rson M.C., Ig64. Studies of the woodland light clinate. I. The photographe computation of light conditions. J. Ecol., $52(\mathrm{I}), 27-4 \mathrm{I}$.

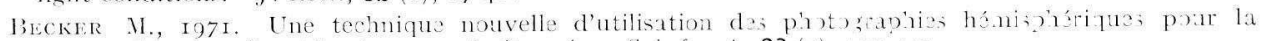
mesure du microclimat lumineux en forêt. Ann. Sci. forest., 23 (4), 425-472.

Bonнomat R., I974. Détermination des profils d'indice folixire et d rayonn ment dans un couvert végétal à l'aide de photographies hémisphériques faites in situ. Th̀̀: 1) octeur-Ingénieur, Univ. Aix-Marseille, C. X. R. S., A. O. 9675 .

Ciark F. C., I96I. An hemispherical forest photocanopymeter. J. Forest, 53, 103-105.

DoGialx R., Ig73. Représentations analytiques des composantes du rayonnenent lumineux solaire. Conditions de ciel serein. Inst. roy. météorol., Belgiqus, Note prélin., $11^{\circ}$ I 7.

Decrey M., ig7o. Utilisation des photographies hémisphériques po:ur l'évaluation de la densité du couvert forestier et pour le calcul de la probéabilité au rayonzaneat solaire. Publ Stn. Syloicult. I. X. R. A.-C. X. R. I., $11^{\circ} 70-4$.

I) TREY M., 1974. Perméabilité des couverts focestiers au rayounensat solaire, appli ation aux photographies hémisphériques, Publ. Stn. Sylvicull, I. N. R. A.-C. N. R. F., $1^{\circ} 74^{-02}$.

Decrey M., I975. Utilisation des photographies hémisphériques po:r la calaul de la perméabilité des couverts forestion an rayounzinzat solair. I. Analys thoriqu de l'interception. Ann. Sci. forest., $32(2), 73-92$.

livass (i.C., Coombe D. E., r959. Hemispherical and wookland canoy photosraphy and light climate, J. Ecol., 47, 103-1 I3.

Ilich R, ig24. A lens for whole sky photographs. Quart. J. roy. moteorol. Soz, 50, 227-235.

Rousser. L., 1953. Recherches théoriques et pratiques sur la répartition en quantité et qualité de la lumière dans le milieu forestier. Influence de la végétation. A nn. E. Nat. Eaur Forêts, 13 (2), 295-385. 